\title{
Simulation Infrastructure Design on the Basis of the Space Industry's International Standards
}

\author{
Ludmila Nozhenkova, Olga Isaeva, Aleksey Markov, Andrey Koldyrev, Rodion Vogorovskiy \\ and Alexander Evsyukov \\ Institute of Computational Modelling of the Siberian Branch of the Russian Academy of Sciences, Akademgorodok 50/44, \\ 660036 Krasnoyarsk, Russia
}

\begin{abstract}
We have designed a technology for creation of the space systems' onboard equipment simulation problem-oriented infrastructure, based on the Simulation Model Portability standard. The simulation infrastructure is a software environment allowing to create, integrate and use simulation models of different purpose including those of different manufacturers'. The infrastructure includes software components, specifications, simulation models and the results of tests, as well as the knowledge bases consolidating knowledge and experience of the experts in space systems' onboard equipment creation. Our product will help to increase the quality and validity of designer solutions at different stages of the space equipment production lifecycle.
\end{abstract}

Keywords-simulation model portability; spacecraft; onboard equipment; command and measuring system; simulation infrastructure

\section{INTRODUCTION}

Unification of space equipment production requires unified approaches to the software tools supporting its production lifecycles. The European Cooperation for Space Standardization has developed a number of standards recommending computer modeling technology for support of all the stages of space projects' realization. [1]. There are simulation models created for different design and production tasks, that provide the necessary characteristics and properties of the space systems. In order to provide construction and usage of complex multi-component simulation models describing the specifics of function of software-hardware systems, it is necessary to increase integrability of the models in complex solutions. In this regard, a topical direction of research is creation of the libraries of reusable models and of their shared use technologies, independently from the software tools applied for their building. [2]. The main principles providing integrability of different manufacturers' models are set by the international Simulation Model Portability (SMP) standard $[3,4]$. The standard determines universal approaches to organization of the simulation systems for simulation models building and their transferability in the modeling environments and operation systems.

In this article we present the product of our research in creation of an original technology for building of a space systems' onboard equipment simulation problem-oriented infrastructure, based on the Simulation Model Portability standard. The technology adds the standard's architectural and functional requirements to software with the original methods of information-graphical and intellectual modeling [5, 6]. The standard sets the general principles of model interface description and regulates functionality of the software modules constituting the core of the modeling infrastructure. However, it doesn't solve a number of problems concerning construction, modeling, integration and interaction of the models, and they are solved by software designers individually. Presently, there is a number of simulation infrastructure designs. The biggest and most significant ones are: SimTG - Astrium Satellites [7], SimSAT - European Space Agency [8], the European mission control center simulator SWARMSIM [9]. Each of the projects is based on the standard, but has different technological approaches in simulation and experiment conduction. The introduced functional additions are aimed at optimal task solution, determined by the purposes of the infrastructure building. We suggest technological and software approaches that allow not only to provide usage of models in different projects and in different programm, but also to consider the specifics of the task under solution. Information-graphical modeling allows to set the system's structure in a graphical form, visualize model's behavior during simulation and the events appearing in it related to the onboard systems' interaction: command transmission, telemetry generation and analysis. The intellectual modeling method allows in an interactive mode to create model rules describing the onboard systems' interaction logics in terms of the subject area. The ability to set the function logics in form of rules allows the constructor to easily build and then modify a simulation model without using programming skills. The technology of simulation infrastructure construction that integrates information-graphical, intellectual and simulation methods and meets the requirements SMP, is an original and promising one for such task solution.

\section{THE TASKS OF THE ONBOARD EQUIPMENT SIMULATION INFRASTRUCTURE}

The functions of our simulation infrastructure are determined by the basic tasks of the space systems' onboard equipment design. The software tasks include support of the design and analysis of the designer solutions at different stages of production lifecycle. These tasks can be technologically presented in actions for which the software can be applied (Figure 1). 


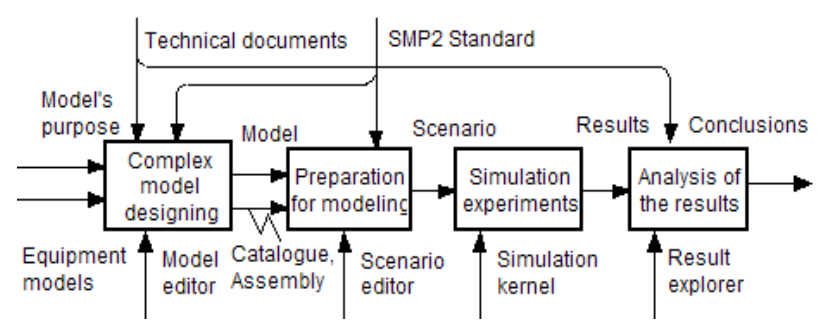

FIGURE I. TASKS OF THE ONBOARD EQUIPMENT SIMULATION INFRASTRUCTURE

A complex model design is performed on the basis of the analysis of the purpose and tasks that need be solved by this simulation model. The structure of the modeled onboard system is built, and its subsystems and their functional dependencies are defined. An onboard equipment designer must be able to build space systems' functional models using the existing models of different manufacturers or creating his own ones in a special environment not requiring additional programming skills from him. The infrastructure provides a rule editor allowing to create model's elements' functions and the logics of their interaction in an interactive mode. This functions will let a specialist to easily build and then modify a simulation model without programming. The rules are clear, thus letting a constructor understand the models, both his own and the ones created by the other specialists, and to see the modeled system in whole. The examples of the space equipment intellectual modeling methods are described in literature [10], but such research does not satisfy the principles of transferability provided by the SMP standard, and they cannot be used in simulation infrastructures. We have developed our own technology of simulation models' integration, including those built on the no-rule basis, and those created in accordance with the SMP standard. The technology allows to build complex models of big systems by uniting the models on the basis of the rules and the SMP models in a one complex model with support their modification. Different realizations of the models are transformed into a unified form by means of the information-graphical modeling tools, and this allows to use the same approaches and software tools for simulation tests preparation and execution, as well as for visualization and analysis of the results of the modeling.

\section{SimUlation MODELING INFRASTRUCTURE REALIZATION}

At the moment the simulation infrastructure is under realization. We are developing the simulation core, graphical modeling tools, editors of models, rules and scenarios. The simulation modeling core includes the mechanisms defined by the SMP standard: Time Keeper, Scheduler, Logger, Event Manager Link Registry, Resolver. The infrastructure includes a subsystem of integration that makes automated interpretation of the models, adding it the graphical structures and mechanisms necessary for the joint work of the models. The modeling core initiates execution of the SMP models and carry out logical inference on the basis of the rules. It allows to manage the speed and the process of the simulation experiment. Its functions include gathering of information about the model's performance during simulation tests, control of the order of messaging between the model's elements and change of the model's condition. The modeling core contains the functions of visualization and saving of the results of modeling, allow to further retrospective analysis of the simulation results. Each of the software subsystems is a special tool providing advanced features for task solution.

High level of the problem orientation in the simulation infrastructure is provided due to model design graphical tools and the rule editor. An example of model element's graphical presentation and knowledge base is given in Figure 2.

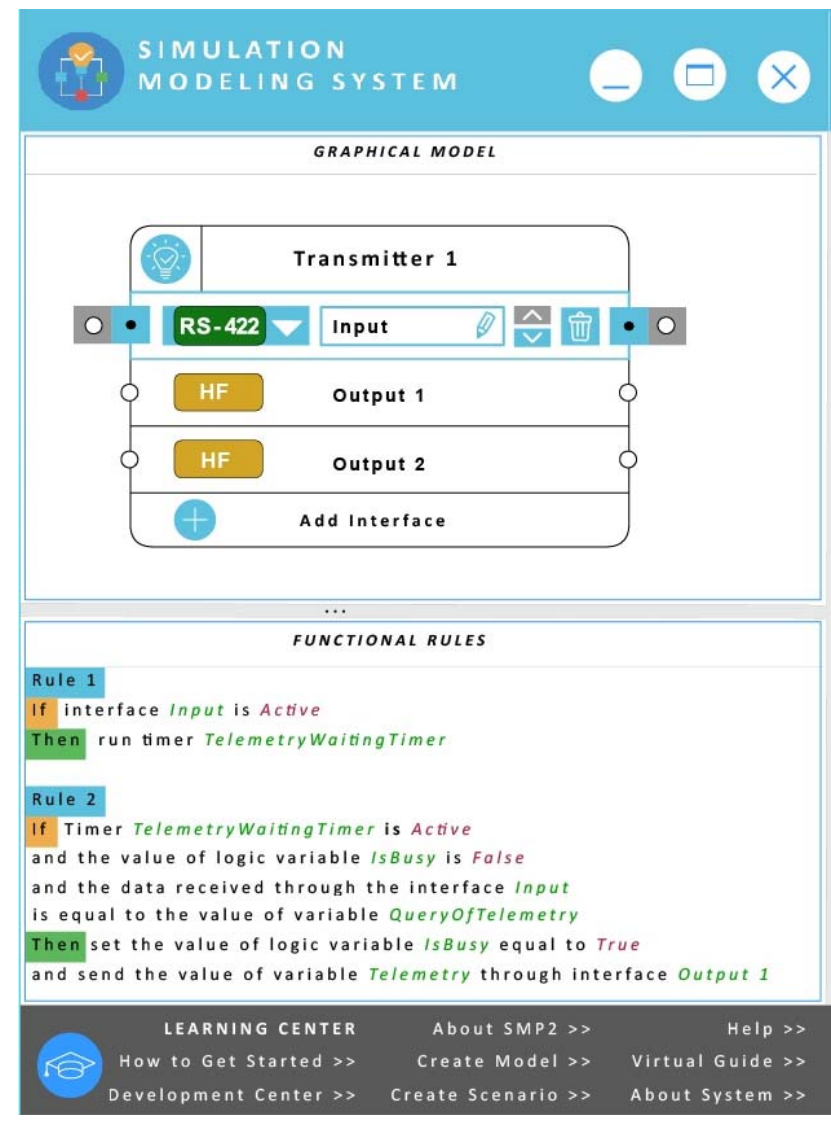

FIGURE II. GRAPHICAL MODELING TOOLS AND RULE EDITOR

The program windows with the model parameters are shown in Figure 3. The tools allow a designer to use familiar semantic constructions to build onboard equipment models of work. The methods are set in form of condition-action rules. Our special tools for knowledge base creation allow to describe different variants of the modeled objects' behavior. These tools were tested earlier, they were used to build function models of spacecraft command-and-measuring system's onboard equipment [11]. Their implementation in the simulation infrastructure will extend the abilities to build models and provide their transferability and integrability in complex solutions. 


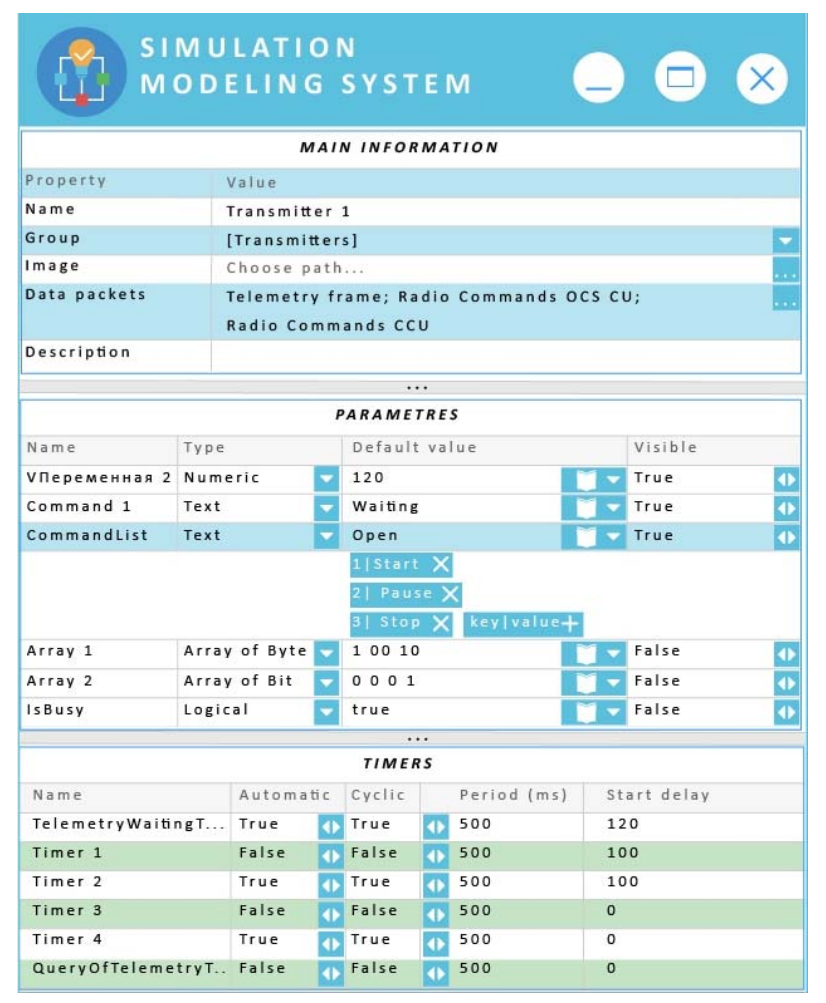

FIGURE III. MODEL PARAMETERS

Figure 4 (at the end of the article) shows the view of the main window of the of infrastructure's for the simulation of spacecraft onboard equipment functions. The navigation tools for the models, scenarios and the results of modeling allow to easily choose, perform and view the ready model solutions. The linkage of the models, scenarios and the results supported by the software, is an end-to-end technology of modeling and analysis of the spacecraft onboard equipment functions.

\section{CONCLUSION}

The technology for build of problem-oriented simulation infrastructure, based on the Simulation Model Portability standard, and it realization allow to build different models of space systems' onboard equipment. Our software includes all the necessary components for simulation models' integrability and transferability support. The software subsystems' structure is added with the original tools of graphic and intellectual modeling that provide creation, conservation and replication of the onboard equipment constructors' unique experience. Usage of the modeling tools will allow to increase the quality and propriety of designer solutions at different stages of space equipment production lifecycle.

Creation of centralized banks of models and modeling scenarios will provide knowledge exchange of specialists of different departments and areas of the instrument making industry, involved in the space systems design.

\section{ACKNOWLEDGMENT}

The reported study was funded by RFBR and Government of Krasnoyarsk Territory according to the research project № 16-41-242042.

\section{REFERENCES}

[1] Space engineering. System modelling and simulation // ESA Requirements and Standards Division ESTEC, 2010.

[2] Lammen W.F., Moelands J., MOSAIC Release 7.1: User Manual, NLRCR-2006-517, NLR 2006.

[3] Simulation modelling platform. ECSS E-40-07 // ESA Requirements and Standards Division ESTEC, The Netherlands, 2011, p. 49.

[4] Simulation Model Portability 2.0 Handbook, 2005, EGOS-SIM-GENTN-0099, issue 1, revision 2, 2005.

[5] Nozhenkova L.F., Isaeva O.S., Gruzenko E.A., Koldyrev A.Yu, Markov A.A., Belorusov A.I., Vogorovskiy R.V. Unified description of the onboard equipment model on the basis of the «Simulation Model Portability» standard // Advances in Intelligent Systems Research (ISSN 1951-6851), Vol. 133, 2016, pp. 481-484, doi:10.2991/aiie-16.2016.111.

[6] Nozhenkova L.F., Isaeva O.S., Gruzenko E.A., Koldyrev A.Yu. Integration technology of the onboard equipment simulation models in simulation modeling infrastructure // Proceedings of the 2016 International Conference on Electrical Engineering and Automation (ICEEA2016), 2016, pp.618-622, doi:10.12783/dtetr/iceea2016/6728.

[7] C. Cazenave and W. Arrouy, "Implementing SMP2 Standard within SimTG Simulation Infrastructure" SESP (ESA): Simulation and EGSE for Space Programmes, 2012.

[8] J. Eggleston, H. Boyer, D. van der Zee, A. Pidgeon, N. de Nisio, F. Burro, and N. Lindman, "Simsat 3.0: Esoc's New Simulation Infrastructure," 6th International Symposium on Reducing the Costs of Spacecraft Ground Systems and Operations (RCSGSO).

[9] Peter Fritzen, Daniele Segneri and Max Pignède, "SWARMSIM - The first fully SMP2 based Simulator for ESOC," 11th Int. WS on Simulation \& EGSE facilities for Space Programmes SESP, 2010.

[10] Eickhoff J. Simulating Spacecraft System. Springer-Verlag Berlin Heidelberg, P. 360, 2009

[11] Nozhenkova L., Isaeva O., Gruzenko E. Computer Simulation of Spacecraft Onboard Equipment // ACSR-Advances in Comptuer Science Research, Vol.18, 2015, pp.943-945, DOI: 10.2991/cisia-15.2015.255. 

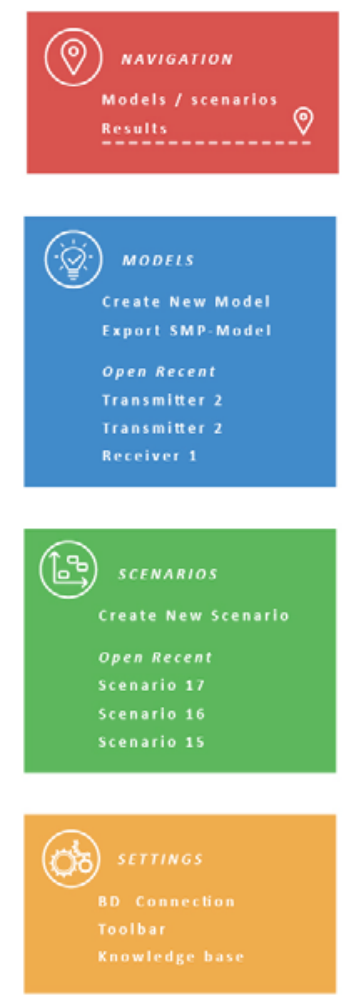

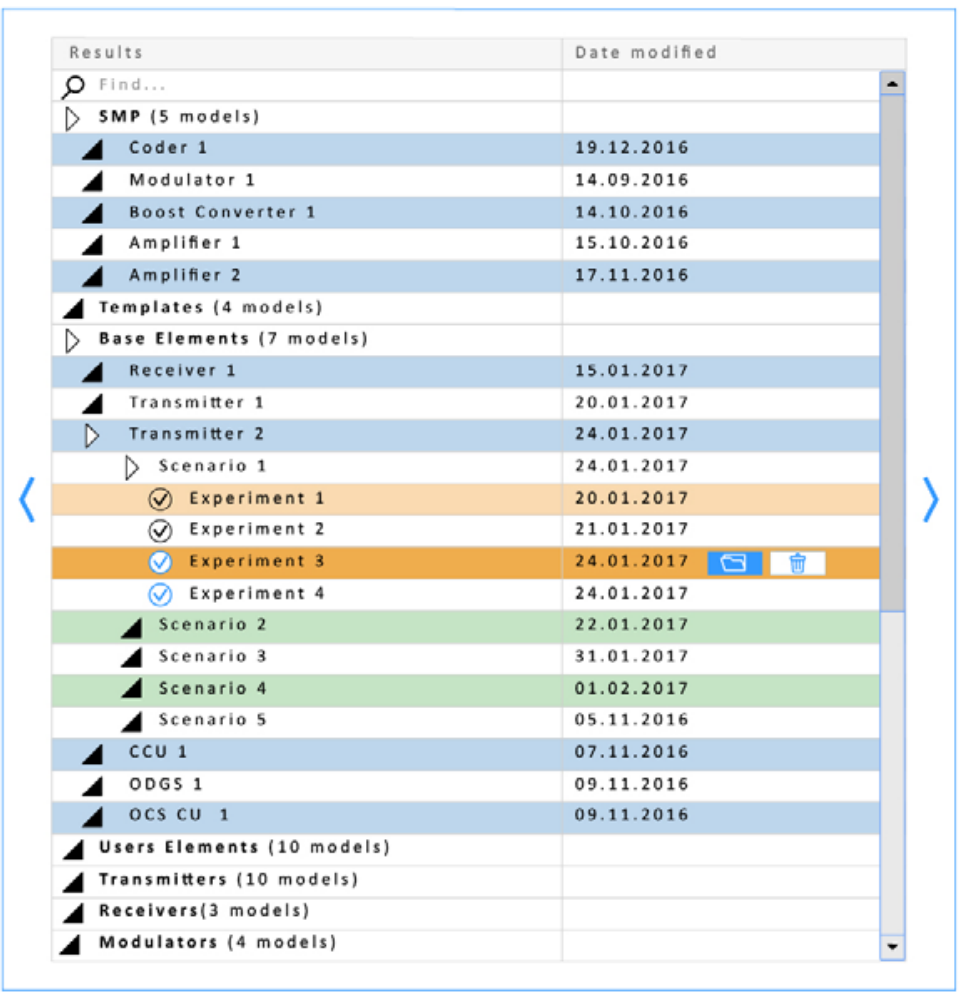

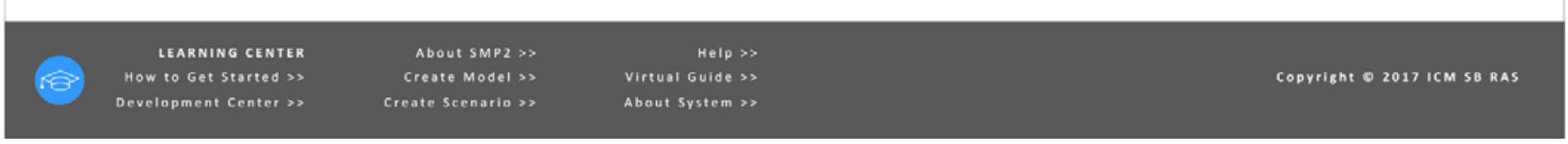

FIGURE IV. THE MAIN WINDOW OF THE OF INFRASTRUCTURE'S FOR THE SIMULATION OF SPACECRAFT ONBOARD EQUIPMENT FUNCTIONS 